\title{
Ein Geben und Nehmen, das alle bereichert - Mobile Hilfe Madagaskar
}

Madagaskar, der flächenmäßig zweitgrößte Inselstaat der Welt, liegt vor der Ostküste Mosambiks im Indischen Ozean und gehört zu den ärmsten Ländern der Welt. 2015 betrug die Rate der unterernährten Menschen 42,3\% und ist damit eine der höchsten der Welt. Die Mobile Hilfe Madagaskar (MHM) hat das Ziel, die Versorgung der Menschen zu verbessern.

Allein die Säuglingssterblichkeit beträgt 42 pro 1000 Lebendgeburten, die Müttersterblichkeit 353 pro 100000 Lebendgeburten. Viele der Frauen sterben während der Geburt auf dem Weg in ein Krankenhaus - denn aufgrund der schlechten Infrastruktur der Insel sind viele Dörfer nicht mit dem Auto zu erreichen und die Menschen sind ausschließlich zu Fuß unterwegs.

Schon der Name „Mobile Hilfe Madagaskar" des Hilfsvereins, den Tanja Hock gemeinsam mit ihrem Mann 2010/2011 gegründet hat, verweist auf die fehlende Mobilität als eines der Hauptprobleme Madagaskars. Tanja Hock kommt aus Aschaffenburg, ist gelernte Hebamme und Rettungssanitäterin. Sie kam 2006 mit einer Schweizer Hilfsorganisation ins Land und ist geblieben.

\section{Hebammenmobil}

Schon bald nach ihrer Ankunft auf Madagaskar machte Tanja Hock die erschütternde Erfahrung, dass Familien häufig Neugeborene im Müll entsorgten, weil Mütter keine rechtzeitige Hilfe bei der Geburt erhielten. Die deutsche Hebamme organisierte daraufhin ein ausgedientes Bankmobil, brachte es beladen mit Hilfsgütern nach Madagaskar und funktionierte es zum „Sage-femmemobile“ um. Inzwischen fährt das Hebammenmobil vier Abende in der Woche durch die Straßen von Antananarivo, der Hauptstadt Madagaskars, und versorgt zahlreiche Menschen, die auf der Straße leben. Neben vier Mitarbeitern (eine Hebamme, ein Arzt, ein Kinderdienstmitarbeiter und ein Fahrer) ist das rollende Krankenhaus mit Strom, Wasser, Klimaanlage und einem Inkubator ausgestattet. Seit 2012 fährt das Hebammenmobil auch in die angrenzenden Dörfer der Hauptstadt, um dort bei jedem Einsatz rund 100 Menschen zu versorgen, die sonst keinen Zugang zu medizinischer Versorgung hätten.

\section{Zahnarztmobil und Krankenwagen}

Schon in den ersten Vereinsjahren wurde klar, dass eine ausschließlich auf Frauen und Kinder ausgerichtete Hilfe nicht durchführbar ist, da auch andere Teile der Bevölkerung Unterstützung benötigen. War der ausgediente Rettungswagen zunächst dazu bestimmt, Notfälle der Mobilen Hilfe
Madagaskar ins nächste Krankenhaus zu transportieren, so wird er heute auch von anderen Helfern genutzt, denn die wenigen krankenhauseigenen Rettungsfahrzeuge in Madagaskar sind schlecht ausgerüstet und haben lange Anfahrtswege.

In die ganz entlegenen Regionen der großen Insel kommt das Team der MHM nur mit dem Flugzeug und so hat der Verein vor einigen Jahren ein Ultraleichtflugzeug angeschafft. Es bringt die Helfer in Dörfer, die von der Außenwelt abgeschnitten sind und keine Krankenstation haben. Seit 2012 fährt zudem ein Zahnarzt-Mobil einmal wöchentlich in ländliche Regionen. Dieses Team hat sich neben der zahnärztlichen Versorgung das Ziel gesetzt, Kindern mit Zahnbürsten und Zahnpasta Zahnhygiene und -prophylaxe beizubringen. Inzwischen werden so rund 1000 Kinder in drei Schulen und in einem Schülerwohnheim versorgt.

\section{Geburtshilfliches Krankenhaus}

Seit Ende 2009 steht in dem kleinen Dorf Ambovo eine Krankenstation, in die Schwangere zur Vorsorge kommen. Tanja Hock hat im Laufe der Jahre einheimische Frauen zu Hebammen ausgebildet, die sich nun von Montag bis Samstag um Vor- und Nachsorge, Familienplanung, Untersuchungen von Neugeborenen und Impfungen 
kümmern. Schon bald waren die Räume zu klein für all die Menschen, die jährlich zur Behandlung kamen (2018 waren es rund 30 000). Tanja Hock wagte größere Träume und mithilfe vieler Spenden wurde im März 2018 der erste Spatenstich für ein geburtshilfliches Krankenhaus gesetzt. Ende des Jahres gewann sie den Leserpreis einer großen deutschen Zeitung und weitere 30000 Euro beschleunigten den Krankenhausbau und die Inbetriebnahme: Schon im Januar 2019 konnte eröffnet werden. Eine stationäre Behandlung wie etwa von Frauen nach schwierigen Geburten oder von Müttern mit Frühgeborenen ist nun möglich. Kaiserschnitte und Notfälle werden von einem Team aus einheimischen und ausländischen Ärzten durchgeführt.

Neben der stationären Versorgung dient das Krankenhaus auch als Trainingszentrum, um einheimische Hebammen und Ärzte weiterbilden zu können. Seit Sommer 2020 baut Tanja Hock mit ihrem Team auch eine kleine Pädiatrie auf, um z. B. Frühgeborene behandeln zu können. Im Vergleich zu den staatlichen Krankenhäusern ist der Betreuungsschlüssel deutlich besser - das spricht sich in der Bevölkerung herum.

\section{Geben und Nehmen}

Neben dem Hebammenmobil, dem Gesundheitszentrum und der Versorgung der Umgebung per Flugzeug ist die MHM ein wichtiger Arbeitgeber geworden. Der Verein beschäftigt in Madagaskar rund 90 Menschen und sichert so vielen Familien ihren Lebensunterhalt. Ein wichtiges Ziel ist die Aus- und Weiterbildung einheimischer Mitarbeiter-ob als Hebammen, Piloten oder Hausmitarbeiter. Auch freiwillige Helfende aus dem Ausland sind willkommen-Hebammen, Zahnärzte, Ärzte, Bürokräfte oder Buchhalter-können „Einblicke in eine ganz andere Welt mit ihren eigenen Stärken und Herausforderungen erleben“, wie es auf der Homepage des Vereins heißt. „Es ist ein Geben und Nehmen, das alle bereichert."

Wer nicht vor Ort helfen, aber einen Beitrag leisten will, kann den Verein mit Spenden unterstützen. „Eine Behandlung kostet 8 Euro, eine Sectio 250 Euro“, berichtet Tanja Hock am Telefon. Damit könne exzellente Arbeit geleistet werden. Auch Patenschaften sind möglich-für eine Schwangere kostet die Behandlung und Vorsorge für 10 Monate 25 Euro. Um einem Kind ein Jahr lang den Schulbesuch zu ermöglichen, sind ebenfalls 25 Euro nötig.

Die Corona-Pandemie beendete im März 2020 die Unterstützung durch ausländische Freiwillige abrupt. Alle Häfen und der internationale Flughafen sind geschlossen. Madagaskar ist nun wirklich eine Insel. Die Arbeit vor Ort hat sich Corona-bedingt nur wenig geändert. Das Hebammen- und das Zahnarztmobil dürfen aber nicht mehr über die Dörfer fahren. Sie stehen nun auf dem dorfeigenen Fußballplatz und werden zur Behandlung von nicht-schwangeren Patienten genutzt, während das Krankenhaus den Müttern und Kindern vorbehalten ist. Die Zahl der einheimischen Mitarbeitenden ist in den jüngsten Monaten erneut gestiegen und es hat sich gezeigt, wie wertvoll die Fortbildung des einheimischen Personals ist. Denn auch ohne die Unterstützung durch ausländische Freiwillige läuft die Arbeit in allen Bereichen der MHM gut.

\section{Katharina Kerlen-Petri}

KONTAKT
Mobile Hilfe Madagaskar e. V.
Im Mathildenweg 22
64832 Babenhausen
Deutschland
Telefon: +49 (0) 1729544243
(per whatsapp)
E-Mail:
info@mobile-hilfe-madagaskar.de
Internet:
www.mobile-hilfe-madagaskar.de
Spendenkonto:
Mobile Hilfe Madagaskar e. V.,
Sparkasse Aschaffenburg,
IBAN: DE05795500000011418472,
BIC: BYLADEM1ASA

